\title{
Optimización del diagnóstico etiológico en niños hospitalizados por empiema pleural y sus beneficios
}

\author{
Optimization of etiologic diagnosis in children hospitalized \\ for pleural empyema and its benefits \\ Elizabeth Assandri,* Claudia Gutiérrez, ${ }^{\ddagger}$ Federica Badía, ${ }^{\S}$ Mónica Pujadas," Inés Mota,, \\ Adriana Varela, ${ }^{* *}$ Karina Machado, ${ }^{\#}$ Ana Paula Méndez, ${ }^{\S \S}$ Eliana Pérez, ${ }^{\S \S}$ Natalia Hermida, ${ }^{\S \S}$ \\ Valeria Le Pera, ${ }^{\text {ๆๆ E}}$ Eduardo Rompani, ${ }^{\S \S}$ M Catalina Pírez, "\| Gabriela Algorta*** \\ * Ex-Profesora Adjunta Clínica Pediátrica A, Departamento Pediatría, FM-UDELAR. \\ ‡ Médico Especialista en Microbiología. Asistente de Microbiología, Departamento de Patología Clínica, FM-UDELAR. \\ § Profesora Adjunta Clínica Pediátrica A, Departamento Pediatría, FM-UDELAR. \\ " Profesora Agregada de Clínica Pediátrica A, Epidemióloga e Infectóloga Pediátrica. FM-UDELAR. \\ " Médico Especialista en Microbiología. Médico, Laboratorio de Microbiología, Departamento de Patología Clínica, CHPR, Administración \\ de los Servicios de Salud del Estado (ASSE). Profesora Adjunta Departamento de Bacteriología y Virología, FM-UDELAR. \\ ** Médico Especialista en Microbiología. Médico, Laboratorio de Microbiología, Departamento de Patología Clínica, CHPR, ASSE. \\ 㭋 Profesora Agregada Clínica Pediátrica A, Departamento Pediatría, FM-UDELAR. \\ \&s Asistentes de Clínica Pediátrica. FM-UDELAR. \\ If Prof. Adj. Pediatría. FM-UDELAR. \\ \|I II Profesora Clínica Pediátrica, A. Departamento de Pediatría. Directora de la Diplomatura de Infectología Pediátrica de FM-UDELAR. \\ *** Médico Especialista en Microbiología. Laboratorio de Microbiología, Departamento de Patología Clínica, CHPR, (ASSE).
}

\section{RESUMEN}

Introducción: El empiema pleural (EP) es una complicación grave de niños con neumonía adquirida en la comunidad (NAC); el cultivo de sangre y/o líquido pleural (LP) confirma la etiología en $5-10 \%$ de los casos de NAC y en $40 \%$ en EP. La incorporación de nuevas técnicas aumenta la probabilidad de confirmación etiológica. Objetivo. General: describir cambios en diagnóstico etiológico y su impacto en la atención de niños con EP. Específicos: A. Describir técnicas microbiológicas. B. Comparar resultados microbiológicos en niños con EP en dos periodos: 1/1/2011-31/12/2017 y 1/1/2018-31/12/2018 (año de inicio de uso sistemático de nuevas técnicas). C. Analizar adecuación del tratamiento según resultados. Material y métodos: Estudio descriptivo. Población: menores de 15 años con EP. Muestras: hemocultivo y LP. Técnicas microbiológicas: 1 . Directo y cultivo. 2. Detección de antígenos (Ags) capsulares en LP. 3. Detección de ácidos nucleicos (AN) en LP y hemocultivos positivos. Fuentes: historias y registros de laboratorio. Resultados: A. Se describen técnicas microbiológicas. B. Resultados microbiológicos. a) 1/1/2011 al 31/12/2017. EP $=211$. Tasa hospitalización/10,000 egresos: 29. Aislamientos bacterianos (cultivo de sangre-LP): 78 (36.9\%): S. pneumoniae 45 casos, otros: H. influenzae, S.

\section{ABSTRACT}

Introduction: Pleural empyema (PE) is a serious complication of children with community-acquired pneumonia (CAP); the culture of blood and / or pleural fluid (PF) confirms etiology in $5-10 \%$ of cases of CAP and $40 \%$ in PE. The incorporation of new techniques increases the probability of etiological confirmation. Objective: Describe changes in aetiological diagnosis and its impact on the care of children with PE. Specific: A. Describe microbiological techniques. B. Compare microbiological results in children with PE in 2 periods: 1/1/2011 to 31/12/2017 and $1 / 1 / 2018$ to $31 / 12 / 2018$ (year of beginning of systematic use of new techniques). C. Analyze treatment adequacy according to results. Material and methods: Descriptive study. Population: under 15 years with PE. Samples: blood culture and PF. Microbiological techniques: 1. Direct smear and culture. 2. Detection of capsular antigens (ags) in LP. 3. Detection of nucleic acids (NA) in PF and positive blood cultures. Sources: clinical and laboratory records. Results: A. Microbiological techniques are described. B. Microbiological results. a) 1/1/2011 to $31 / 12 / 2017$. $P E=211$. Hospitalization rate/10,000 discharges: 29. Bacterial isolates (blood/PF cultures): 78 (36.9\%): S. pneumoniae 45 cases, Others: H. influenzae, S. pyogenes, S.

Citar como: Assandri E, Gutiérrez C, Badía F, Pujadas M, Mota I, Varela A et al. Optimización del diagnóstico etiológico en niños hospitalizados por empiema pleural y sus beneficios. Rev Latin Infect Pediatr. 2021; 34 (3): 120-127. https://dx.doi.org/10.35366/102234

Recibido: 11-01-2021. Aceptado: 25-06-2021.

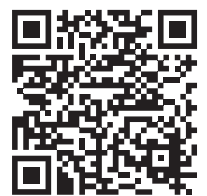


Rev Latin Infect Pediatr. 2021; 34 (3): 120-127

pyogenes, S. aureus, anaerobios, S. mitis y M. tuberculosis. Casos con bacteria identificada agregando identificación esporádica por Ags y AN: 64\%; S. pneumoniae 102. b) 1/1/2018 al 31/12/2018. $\mathrm{EP}=33$, tasa $34 / 10,000$ egresos. Etiología por cultivo, Ags y AN: 29 (87.8\%). S. pneumoniae 26. Otros: H. influenzae, S. pyogenes, K. pneumoniae. Proporción de aumento en identificación etiológica $37.8 \%(p=0.03)$. C. Adecuación antimicrobiana: a) 1/1/2011 a 31/12/2017: $90.4 \%$ de casos. b) 2018: $94.4 \%$. Conclusiones: Se logró optimizar el diagnóstico etiológico en niños con EP, lo que tiene impacto en la antibioticoterapia y aporta información para la elaboración de pautas de tratamiento empírico en los casos de empiema pleural.

Palabras clave: Empiema pleural, diagnóstico etiológico, niños.

\section{INTRODUCCIÓN}

El empiema pleural (EP) es una complicación grave de los niños con neumonía adquirida en la comunidad (NAC). Si bien el número de casos ha descendido significativamente desde la incorporación universal de vacunas conjugadas, esta patología continúa generando importante morbilidad. ${ }^{1-3}$

Desde el año 2008, año en que se inicia en Uruguay la vacunación universal con vacuna neumocócica 7 valente (sustituida en 2010 por 13 valente), se produjo una disminución significativa de los casos de NAC y NAC complicada causados por S. pneumoniae. Dada esta diminución de casos por $S$. pneumoniae, la proporción de otros agentes etiológicos como causantes de estas entidades clínicas es mayor. Dentro de estos agentes se encuentran: $S$. aureus meticilino resistente adquirido en la comunidad (SAMR-AC) o meticilino sensible (SAMS), $H$. influenzae no tipificable o $H$. influenzae tipo b, $S$. pyogenes y $M$. tuberculosis. Éstos han pasado a representar alrededor de $30 \%$ de los casos en que se confirma etiología en niños con EP, generando entonces un desafío a la hora de decidir el tratamiento antibiótico inicial. .-9 $^{-9}$

La demora en instaurar un tratamiento adecuado puede llevar incluso a la muerte del paciente. Por otra parte, el uso racional de antibióticos resulta fundamental. La morbilidad y prolongada evolución que pueden presentar estos niños con neumonía complicada puede llevar al clínico al uso de múltiples antibióticos, a veces en forma innecesaria. ${ }^{3,8-15}$ Es por eso que la identificación bacteriana cobra importancia en cada caso individual. Además, la identificación etiológica permite conocer la epidemiología local y nacional. Esta información es fundamental para establecer pautas de tratamiento empírico inicial como las recomendadas en Uruguay desde 1997 aureus, anaerobes, S. mitis and M. tuberculosis. Cases with identified bacteria adding sporadic identification by ags and NA: $64 \%$; S. pneumoniae 102 . b) $1 / 1 / 2018$ to $31 / 12 / 2018$. $P E=33$. rate 34/10,000 discharges. Etiology by culture, ags and NA: 29 (87.8\%). S. pneumoniae 26. Others: H. influenzae, S. pyogenes, K. pneumoniae. Proportion of increase in etiological identification $37.8 \%(p=0.03)$. C. Antimicrobial adequacy a) $1 / 1 / 2011$ to 31/12/2017: $90.4 \%$ of cases. b) 2018: $94.4 \%$. Conclusions: The etiological diagnosis was optimized in children with PE. This has an impact on antibiotic therapy, provides information for developing empirical treatment guidelines in children with pleural effusion.

Keywords: Pleural empiema, etiological diagnosis, children.

y actualizadas periódicamente. ${ }^{4,16,17}$ La vigilancia epidemiológica aporta además valiosa información necesaria para la toma de decisiones en cuanto al uso e incorporación de vacunas. . $^{7,8,18,19}$

Con las técnicas habituales de cultivo de sangre y/o líquido pleural se llega a confirmar la etiología en alrededor de 5 a $10 \%$ de los casos de NAC. Al utilizar estas técnicas en los casos de niños con empiema pleural, el porcentaje puede aumentar a alrededor de $40 \%$. $^{7,8,20,21}$

Son muchos los factores que determinan que los microorganismos no estén viables y no se aíslen en los medios de cultivo habituales, entre ellos se encuentra el uso frecuente de antimicrobianos previo a la toma de las muestras, la labilidad de los agentes involucrados y la posibilidad de un manejo inadecuado de las muestras..$^{20,21}$

La incorporación de nuevas técnicas, como la detección de antígenos o de ácidos nucleicos, aumenta la probabilidad de confirmar la etiología y disminuye los tiempos diagnósticos, brindando información oportuna para optimizar el tratamiento antimicrobiano con todos los beneficios que esto conlleva. ${ }^{20,21}$

Con base en lo mencionado previamente, la presente investigación tiene el objetivo de describir los cambios en el diagnóstico etiológico de EP y su impacto en la atención de los niños con esta patología en el Hospital Pediátrico del Centro Hospitalario Pereira Rossell (HP-CHPR), hospital pediátrico de referencia en Uruguay.

Los objetivos específicos son:

A. Describir las técnicas utilizadas en el laboratorio de microbiología para búsqueda etiológica (aislamiento, identificación, detección de antígenos o ácidos nucleicos de los agentes bacterianos) en los casos de NAC y EP. 
B. Comparar los resultados microbiológicos en niños con EP en dos periodos: del 1 de enero de 2011 al 31 de diciembre de 2017 y del 1 de enero de 2018 al 31 de diciembre de 2018. En el año 2018 se comenzaron a utilizar en forma sistemática las nuevas técnicas diagnósticas.

C. Analizar la adecuación del tratamiento antimicrobiano según los resultados microbiológicos en los dos periodos.

\section{MATERIAL Y MÉTODOS}

Se trata de un estudio descriptivo. La población incluye a todos los menores de 15 años ingresados por EP, entre el 1 de enero de 2011 y el 31 de diciembre de 2018 en el HP-CHPR.

Para el diagnóstico de los casos de EP se utilizó la definición de las pautas nacionales, entendiendo por tal a la existencia de una neumonía con derrame pleural, con la presencia en el LP de un criterio mayor: pus y/o evidencia de bacterias observadas en el directo, cultivo o detección de antígenos capsulares; y/o dos criterios menores: glucosa menor o igual a $40 \mathrm{mg} / \mathrm{dL}, \mathrm{pH}$ menor o igual a 7.10, lactato deshidrogenasa (LDH) mayor o igual a 1,000 UI/L, leucocitos en un número mayor o igual a $10 / \mathrm{mm}^{3}$ y $90 \%$ o más de polimorfonucleares.

Las muestras utilizadas para la búsqueda etiológica fueron sangre y líquido pleural.

Las técnicas microbiológicas empleadas fueron: 1. Examen directo y cultivo de sangre o líquido pleural. 2. Detección de antígenos (Ags) capsulares para $S$. pneumoniae y $H$. influenzae tipo b en LP. 3. Detección de ácidos nucleicos (AN) de $S$. pneumoniae y $\mathrm{H}$. influenzae en todas las muestras de LP. 4. En las muestras de hemocultivo que el sistema de incubación detectó como positivas, además del aislamiento en medio de cultivo sólido, se utilizó la técnica de reacción en cadena de la polimerasa en tiempo real (RT-PCR FilmArray ${ }^{{ }_{-}}$ Biomerieux).

Cuando por alguna de las técnicas se detectó algún patógeno, el caso se consideró empiema pleural con etiología confirmada.

Para valorar la adecuación terapéutica se consideraron sólo los casos en los que se obtuvo identificación bacteriana, y dentro de este grupo aquéllos en los que el o los antibióticos utilizados inicialmente eran de mayor espectro antimicrobiano que el necesario para la o las bacterias identificadas. Se consideró en estos casos que se realizó adecuación terapéutica si luego de identificado el agente bacteriano, se adecuó el tratamiento antimicrobiano.

Las fuentes de datos fueron las historias clínicas y los registros del laboratorio de microbiología.

El análisis estadístico se realizó empleando distribución de frecuencias, medidas de resumen (de tendencia central y de dispersión), tasa de hospitalización por 10,000 egresos (IC 95\%) y pruebas de significancia estadística cuando correspondiera (significativo $\mathrm{p}$ menor o igual a 0.05 ). Para la asociación entre variables se utilizó además el test de $\chi^{2}$, los programas estadísticos empleados fueron Microsoft Excel, SPSS y EPI INFO7 ${ }^{\mathrm{TM}}$.

\section{RESULTADOS}

\section{A. Las técnicas utilizadas para búsqueda etioló-} gica en los casos de NAC y EP se describen a continuación:

1. Métodos habituales de observación microscópica (frotis con coloración de Gram) y cultivo en medios adecuados (agar sangre y chocolate incubados en atmósfera enriquecida en $5 \%$ de $\left.\mathrm{CO}_{2}\right)$.

2. Detección de antígenos capsulares solubles por aglutinación de partículas de látex para $S$. pneumoniae y $\mathrm{H}$. influenzae tipo b en líquido pleural.

Se utiliza la técnica de aglutinación de partículas de látex de antígenos capsulares descrita y comercializada para líquido cefalorraquídeo, Pastorex ${ }^{\text {TM }}$ Meningitis Bio-Rad, Marnes-laCoquette, France.

3. Detección de ácidos nucleicos (reacción en cadena de la polimerasa en tiempo real, RT-PCR) en muestras de LP utilizando el panel de meningoencefalitis de FilmArray ${ }^{\circledR}$ - Biomerieux.

Tanto para los antígenos capsulares como para la detección de ácidos nucleicos no hay una metodología aprobada para su uso en líquido pleural, se aplican las diseñadas para meningoencefalitis en las muestras de LP en forma experimental, con las precauciones debidas. Se busca la detección de $S$. pneumoniae y Haemophilus influenzae tipo b para los antígenos y S. pneumoniae y Haemophilus influenzae para la detección de ácidos nucleicos.

4. Detección de ácidos nucleicos en muestras de hemocultivo positivas (reacción en cade- 
na de la polimerasa en tiempo real, RT-PCR) utilizando el panel de bacteriemias de FilmArray $^{\circledR}$ - Biomerieux. Este panel permite detectar ácidos nucleicos de 24 patógenos y tres genes de resistencia (Enterococcus, Listeria monocytogenes, S. aureus, S. agalactiae, S. pyogenes, S. pneumoniae, Acinetobacter baumannii, $H$. influenzae, $N$. meningitidis, $P$. aeruginosa, Enterobacter cloacae complex, E. coli, Klebsiella oxytoca, K. pneumoniae, Serratia marcescens, Proteus, Candida albicans, C. glabrata, C. krusei, C. tropicalis, C. parapsilosis. Genes asociados a mecanismos de resistencia: mec A- meticilino resistencia, Van A/B resistencia a vancomicina, KPC resistencia a carbapenemes).

Los resultados de estas técnicas, que pueden obtenerse en menos de dos horas, permiten acortar hasta en 48 horas el tiempo del informe etiológico mientras se espera el resultado de los cultivos.

Cuando existe sospecha clínica de enfermedad tuberculosa se realiza: baciloscopía, RT-PCR para detección conjunta de micobacterias del complejo tuberculosis y resistencia a rifampicina 5 (GeneXpert®-Cepheid) y se envía parte de la muestra al laboratorio de la CHLA (Comisión Honoraria de Lucha Antituberculosa) para cultivo. Los resultados de estas técnicas también se obtienen rápidamente.
Las cepas de $S$. pneumoniae recuperadas de sangre y/o LP son enviadas al Departamento de Laboratorios de Salud Pública (DLSP) para su tipificación.

Los estudios de susceptibilidad a antimicrobianos se realizan siguiendo las recomendaciones del Clinical and Laboratory Standards Institute, 2019. ${ }^{22}$

El estudio de observación directa y cultivo se utilizó en $100 \%$ de las muestras en los dos periodos; la detección de antígenos capsulares y ácidos nucleicos se realizó esporádicamente (de acuerdo a disponibilidad de reactivos) en el primer periodo (del 1 de enero de 2011 al 31 de diciembre de 2017) y una o ambas técnicas en $100 \%$ de las muestras en el segundo (del 1 de enero de 2018 al 31 de diciembre de 2018).

\section{B. Resultados microbiológicos en niños con EP.} El número de casos hospitalizados por EP así como el número de aislamiento bacteriano por cultivos e identificación por otras técnicas microbiológicas en los dos periodos considerados se muestran en la Tabla 1.

Entre el 1 de enero de 2011 y el 31 de diciembre de 2017 S. pneumoniae fue aislado por cultivo de sangre y/o LP en 45 casos. Todos fueron susceptibles a penicilinas y cefalosporinas. De los aislamientos, $30 \%$ presentaron algún grado de

\begin{tabular}{|c|c|c|}
\hline & \multicolumn{2}{|c|}{ Periodo } \\
\hline & 2011-2017 & 1/1/2018 al 31/12/2018 \\
\hline \multicolumn{3}{|l|}{ Casos de EP } \\
\hline $\mathrm{n}(\%)$ & 211 & 33 \\
\hline Tasa anual de hospitalización/10,000 egresos & $29 / 10,000$ & $34 / 10,000$ \\
\hline IC de $95 \%$ & $18.0-39.0$ & $22-45$ \\
\hline \multicolumn{3}{|l|}{ Aislamiento bacteriano (cultivo de sangre y/o LP) } \\
\hline $\mathrm{n}(\%)$ & $78(36.9)$ & $6(18.1)$ \\
\hline Tasa/10,000 egresos & Tasa $10 / 10,000$ & $6 / 10,000$ \\
\hline IC de $95 \%$ & $4-16$ & $1-11$ \\
\hline \multicolumn{3}{|l|}{ Identificación bacteriana (cultivos + Ags o AN) } \\
\hline $\mathrm{n}(\%)$ & $135(64.0)$ & $29(87.8)$ \\
\hline Total N (\%) & 244 & $84(34.4)$ \\
\hline
\end{tabular}


Tabla 2: Bacterias identificadas por cultivos, detección de antígenos capsulares y ácidos nucleicos en un hospital pediátrico de referencia. 2011-2018. Uruguay.

\begin{tabular}{lrc} 
& \multicolumn{2}{c}{ Periodo } \\
\cline { 2 - 3 } \multicolumn{1}{c}{ Bacteria identificada } & $2011-2117$ & $31 / 01 / 2018-$ \\
\cline { 2 - 3 } S. pneumoniae & 100 & 25 \\
S. pneumoniae + H. influenzae & 2 & 1 \\
S. aureus & 6 & - \\
H. influenzae & 15 & 1 \\
S. pyogenes & 6 & 1 \\
Anaerobios & 1 & - \\
S. mitis & 1 & - \\
M. tuberculosis & 2 & - \\
K. pneumoniae & - & 1 \\
\hline Número de identificaciones & 133 & 29 \\
bacterianas & &
\end{tabular}

resistencia a otros antimicrobianos (eritromicina, trimetoprima-sulfametoxazol o clindamicina).

De las 17 cepas de $H$. influenzae identificadas por cultivo, $20 \%$ eran productoras de $\beta$ lactamasas. De los seis casos de $S$. aureus aislados, cinco correspondieron a $S$. aureus meticilino resistente y uno a $S$. aureus meticilino sensible.

Considerando además de los cultivos la detección de antígenos por aglutinación de látex y el estudio molecular (PCR) realizados en forma esporádica esos años, el número de EP con etiología identificada asciende a $64 \%$ de los casos a expensas de la identificación de 57 casos más de S. pneumoniae.

En ese periodo, por detección de antígenos y/o ácidos nucleicos, no se identificaron casos por otras bacterias distintas a S. pneumoniae.

Considerando todas las técnicas diagnósticas utilizadas en ese periodo, S. pneumoniae fue identificado en 102 casos (representando $75.5 \%$ de los agentes identificados y $48.3 \%$ del total de egresos).

El número de casos de EP con identificación bacteriana así como las bacterias identificadas en el periodo comprendido entre el 1 de enero de 2018 y el $\mathbf{3 1}$ de diciembre de 2018 se muestran en las Tablas 1 y 2 respectivamente. En cuatro casos no se identificaron bacterias. Los estudios microbiológicos de tres de estos pacientes no fueron realizados en el laboratorio del centro donde se llevó a cabo la presente investigación, dado que estos niños fueron asistidos en unidades de cuidados intensivos ( $\mathrm{UCl}$ ) externas en las primeras semanas de su enfermedad. Todos los casos de S. pneumoniae fueron susceptibles a penicilinas y cefalosporinas. De los dos casos de $H$. influenzae hallados, uno fue por cultivo y no era productor de $\beta$ lactamasas. La otra identificación fue por RT-PCR. El promedio de tiempo de comunicación de los resultados microbiológicos en el año 2018 fue de 19 horas (rango se seis a 48 horas).

Comparando los resultados microbiológicos, la proporción de aumento en identificación etiológica en los dos periodos fue de $37.8 \%(p=0.03)$.

\section{Adecuación del tratamiento antimicrobiano} luego de recibido el resultado microbiológico.

En el periodo de 2011 a 2017 en 107 de los 211 casos de EP, la ampicilina no fue el antimicrobiano con el que se inició el tratamiento. En 57 de estos casos se consideró que el paciente presentaba una forma severa de la enfermedad, tres eran pacientes no vacunados y en dos se consideró un mecanismo aspirativo. En 45 casos no se encontró justificación.

Si consideramos la adecuación del antibiótico una vez recibido el resultado microbiológico, esto se produjo en $90.4 \%$ de los casos (mayoritariamente $S$. pneumoniae). Las justificaciones en los 17 casos en los que no se adecuó el antimicrobiano, fueron las siguientes: tres pacientes cursaron enfermedad grave con una estadía muy prolongada en la $\mathrm{UCl}$, dos presentaron coinfección, otro tuvo parénquima durante su internación (una infección de herida quirúrgica en el lugar de inserción del tubo de drenaje y una infección urinaria). En los otros seis pacientes no se encontró justificación.

En el año 2018, el antibiótico inicial no fue ampicilina en $60 \%$ de los casos. De los 20 casos en que el tratamiento inicial no fue ampicilina, se identificó bacteria en 18 de ellos y se realizó la adecuación del antimicrobiano en 17 (94.4\%). No se adecuó en un niño con enfermedad severa con larga estadía en cuidados intensivos.

\section{DISCUSIÓN}

Si bien en Uruguay debido a múltiples medidas de salud pública se logró disminuir significativamente la incidencia de la NAC y NAC complicada, éstas 
Rev Latin Infect Pediatr. 2021; 34 (3): 120-127

continúan siendo una causa importante de ingreso hospitalario. ${ }^{7,8,18}$ En otros países de la región de las Américas éste es también un problema de salud que concita mucha atención. ${ }^{23-25}$ El empiema pleural es una de estas complicaciones. Éste se produce cuando en el derrame pleural paraneumónico se replican bacterias, las mismas pueden ser identificadas por estudio directo o cultivos del LP, o quedar en evidencia por la presencia de pus o alteraciones citoquímicas que se generan por la presencia bacteriana en el LP. ${ }^{16,17,26}$

Identificar la existencia de bacterias o sus componentes en el LP constituye una herramienta diagnóstica en el caso de NAC con derrame. Determinar la etiología en estos casos es fundamental también para el adecuado tratamiento de estos niños. ${ }^{23,26}$

A partir del año 2018 las técnicas de detección de antígenos y/o ácidos nucleicos de los agentes bacterianos se aplican en forma sistemática para la búsqueda etiológica en todos los casos de NAC y NAC complicada asistidos en el HP-CHPR.

La incorporación de estas nuevas técnicas y su aplicación al $100 \%$ de las muestras ha tenido un impacto significativo en la identificación del agente etiológico en niños con EP. La identificación pasó de ser de $36.9 \%$ utilizando los métodos tradicionales (directo y cultivo) a $87.8 \%$ en el año 2018 agregando al directo y cultivo la utilización de las nuevas técnicas ya descritas.

Es de destacar que, de los casos de EP en los que los estudios microbiológicos se realizaron en el laboratorio del CHPR en el segundo periodo, sólo en uno no se identificó agente bacteriano. En los otros tres casos de EP, en los que no se identificó bacteria, de los 33 de ese periodo, los estudios microbiológicos no se realizaron en el CHPR como ya fue mencionado.

En ambos periodos $S$. pneumoniae fue la bacteria más frecuentemente identificada y en ambos no se halló resistencia a penicilinas ni a cefalosporinas. Las cepas aisladas de S. pneumoniae en este centro hospitalario, como en el resto del país, no generan un problema terapéutico. 8,17

Otras bacterias descritas en la literatura como causantes habituales de EP fueron identificadas en el periodo de 2011 a 2017. Entre ellas, en orden decreciente de frecuencia se aislaron: $H$. influenzae, $S$. pyogenes, $S$. aureus meticilino resistente y $S$. aureus meticilino sensible. En dos casos se identificó M. tuberculosis como causante de EP, hecho que no es habitual. Otras bacterias que no son descritas habitualmente como causantes de EP y que se aislaron en el periodo de 2011 a 2017 fueron: S. mitis en un caso y anaerobios en otro (se trató de un niño en el que luego se diagnosticó una malformación pulmonar).

En el segundo periodo no se identificaron casos de EP por $S$. aureus ni por M. tuberculosis. Si bien en ese periodo no se identificaron casos de EP por M. tuberculosis, en el contexto epidemiológico actual, con una reemergencia de esta micobacteria, ${ }^{27-30}$ ésta y otras etiologías distintas a neumococo deben considerarse, particularmente en aquellos pocos casos en los que no se identifica agente causal.

En 2018 como agente inusual de EP se identificó Klebsiella pneumoniae en un cultivo de LP.

Si bien la identificación etiológica en los casos de EP fue significativamente mayor en el segundo periodo analizado, el porcentaje de aislamiento bacteriano por cultivos fue menor en ese periodo. No pudimos identificar la causa, pensamos podría deberse al hecho de que muchos de los pacientes con EP al ingreso hospitalario ya estaban recibiendo antimicrobianos.

La utilización de las nuevas técnicas también redujo el tiempo de obtención de los resultados microbiológicos. Como se mencionó, el promedio de comunicación de estos resultados en el segundo periodo fue de 19.5 horas (rango de seis a 48 horas). Si bien no contamos con datos exactos de estos tiempos en el primer periodo para poder establecer una comparación, sabemos que estos tiempos representan una mejoría significativa.

La identificación del agente etiológico, así como la mejora en el tiempo de obtención de los resultados racionaliza los recursos terapéuticos utilizados y optimiza el manejo integral de estos pacientes..$^{18,26,30}$

El aumento de la identificación etiológica en el segundo periodo se acompañó también de un aumento del porcentaje de casos de adecuación antimicrobiana.

La vigilancia de la etiología de las NAC y NAC complicada y de la susceptibilidad bacteriana es fundamental también para poder realizar actualizaciones de las recomendaciones de tratamiento empírico de estas patologías, según los cambios que se van produciendo; dado que se contribuye al uso racional de antibióticos, es una acción fundamental en la estrategia contra la resistencia antimicrobiana. 
La determinación etiológica es importante también para la toma de decisiones a la hora de incorporar vacunas.

Por todo lo antes dicho, es imprescindible continuar avanzando en la mejora y utilización de todas las herramientas posibles para el diagnóstico etiológico oportuno.

\section{CONCLUSIÓN}

Se logró optimizar el diagnóstico etiológico en niños con EP con un aumento significativo en la identificación bacteriana. Esto tiene impacto en el uso racional y adecuación de los antimicrobianos empleados así como en el manejo integral de estos niños. Aumentar el diagnóstico etiológico en los casos de EP agrega información imprescindible para la elaboración de pautas de tratamiento empírico así como para la toma de decisiones para la incorporación de vacunas. ${ }^{8}$

\section{REFERENCIAS}

1. UNICEF/WHO, Pneumonia: The forgotten killer of children, 2006 ISBN-13: 978-92-806-4048-9 ISBN-10: 92806-4048-8.

2. Boletín epidemiológico semanal. Vacunas antineumocócicas. Documento de posición de la OMS2012 [6 de abril de 2012], año 87 N. 14, 2012, 87, 129-144. Disponible en: http://www.who.int/wer

3. Villena GV, Cases VE, Fernández VA, de Pablo GA, Pérez RE, Porcel PJM et al. Recommendations of diagnosis and treatment of pleural effusion. Update/Arch Bronconeumol. 2014; 50 (6): 235-249A.

4. Pírez MC, Martínez O, Ferrari AM, Nairac A, Montano A, Rubio I et al. Standard case management of pneumonia in hospitalized children in Uruguay, 1997-1998. Pediatr Infect Dis J. 2001; 20: 283-289.

5. Pírez MC, Berrondo C, Giacometti M, Demiguel M, Pascale I, Algorta $\mathrm{G}$ et al. Neumonía bacteriana adquirida en la comunidad en niños hospitalizados. Arch Pediatr Urug. 2003; 74 (1): 6-14.

6. Ferrari AM, Pírez MC, Martínez A, Algorta G, Chamorro $F$, Guala MJ et al. Etiología de la neumonía bacteriana adquirida en la comunidad en niños hospitalizados. Uruguay 1998-2004. Rev Chil Intect. 2007; 24 (1): 45-52.

7. Pírez MC, Algorta G, Cedres M, Sobrero H, Varela A, Giachetto $G$ et al. Impact of universal pneumococcal vaccination on hospitalizations for pneumonia and meningitis in children in Montevideo, Uruguay. The Pediat Infect Dis J. 2011; 30 (8): 669-674.

8. Pírez MC, Algorta G, Chamorro F, Romero C, Varela A, Cedres $\mathrm{A}$ et al. Changes in hospitalizations for pneumonia after universal vaccination with pneumococcal conjugate vaccines $7 / 13$ valent and $H$. influenzae type $b$ conjúgate vaccine in a pediatric referral hospital in Uruguay. Pediatr Infect Dis J. 2014; 33 (7): 753-759.

9. Badía F, Assandri E, Pujadas M, Machado MK, Varela A, Gutierrez C et al. Hospitalizaciones por empiema paraneumónico en un Hospital Pediátrico de Referencia entre los años 2005 y 2016. 3110 Congreso Uruguayo de Pediatría, Montevideo octubre 2017.

10. Pírez M, Giachetto G, Romero R, Zabala C, Algorta G, Montano $A$ et al. Neumonía neumocócica invasiva en niños de 0 a 24 meses: ¿influye la resistencia bacteriana en la evolución? An Pediatr (Barc). 2008; 69 (3): 205-209.

11. Machado M, Gariné Kouyoumdjian, Algorta G, Pírez MC. Neumonía necrotizante en niños hospitalizados en el Hospital Pediátrico del Centro Hospitalario Pereira Rossell en el año 2010. Arch Pediatr Urug. 2013; 84 (2): 101-110, 2013, 101-110.

12. Noya N, Amarillo P, Pérez R, Arana M, Machado K, Dall Orso $\mathrm{P}$ et al. Cambios en las hospitalizaciones por neumonía necrotizante después de la vacunación universal con vacuna antineumococcica conjugada en un hospital de referencia pediátrico. IV Congreso Internacional de Infectología Pediátrica y Vacunas. SADIP. Marzo 2014.

13. González G, Alonso D, Vázquez P, Amaya G, Badia F, Assandri $E$ et al. Neumonía neumococcica necrotizante: identificación de factores de asociación en niños. K machado, 30 Congreso Uruguayo de Pediatría. 8 al 11 de Setiembre 2015.

14. Chamorro F, Amaya G, Badia F, Machado K, Pujadas M, Gutierrez S et al. Neumonia por Staphylococcus aureus meticilino resistente adquirido en la comunidad presentación clínica y evolutiva de niños internados en el Centro Hospitalario Pereira Rossell. Años 2003 al 2014. Congreso 100 años SUP Montevideo. Octubre 2014.

15. Vicudo L, Assandri E, Badia F, Machado K, Pujadas M, Gutiérrez $\mathrm{C}$ et al. Hospitalizaciones por neumonía adquirida en la comunidad causadas por Streptococcus pyogenes en el Centro Hospitalario Pereira Rossell, Uruguay. Periodo 2013-2015. V Congreso Internacional de Infectología Pediátrica y Vacunas. Sociedad Argentina de Infectología Pediátrica. Buenos Aires 5 y 6 de mayo 2016.

16. Pírez MC, Montano A, Rubio I et al. Neumonía. En: Atención pediátrica: pautas de diagnóstico, tratamiento y prevención. 6a ed. Montevideo: Oficina del Libro FEFMUR; 2007.

17. Giachetto G, Pérez W, Pírez MC, Prego J et al. Atención pediátrica: normas nacionales de diagnóstico, tratamiento y prevención. 8a ed. Montevideo: Oficina del Libro FEFMUR; 2014.

18. García GG, López VM, Pérez GG, Hernández S, Cardinal P, Félix $V$ et al. Effect of pneumococcal conjugate vaccination in Uruguay, a middle-income country. PLoSOne. 2014; 9 (11): e112337. doi: 10.1371/journal.pone.0112337.

19. Montano A, Algorta G, Pírez MC, Farcilli R, Pascale G, Ferrari AM. Enfermedades invasivas por Haemophilus ilnfluenzae tipo b. Impacto de la vacunación en los niños que ingresan al Centro Hospitalario Pereira Rossell. Rev Med Uruguay. 2001; 17 (3): 166-170.

20. Stankey CT, Spaulding AB, Doucette A, Hamre KES, Wheeler W, Pomputius WF et al. Blood culture and pleural fluid culture yields in pediatric empyema patients: a retrospective review. 1996-2016. Pediatr Infect Dis J. 2018; 37 (9): 952-954. doi: 10.1097/ INF.0000000000001940.

21. Study Group. Collaborators: O'Brien KL, Baggett HC, Brooks WA, Feikin DR, Hammitt LL, y colaboradores. Causes of severe pneumonia requiring hospital admission in children without HIV infection from Africa and Asia: the PERCH multi- 
Rev Latin Infect Pediatr. 2021; 34 (3): 120-127

country case-control study. Pneumonia Etiology Research for Child Health (PERCH). Lancet. 2019; 394 (10200): 757779. doi: 10.1016/S0140-6736(19)30721-4.

22. CLSI. Performance Standards for Antimicrobial Susceptibilty Testing. 29th ed. CLSI supplement M100. Wayne, PA: Clinical and Laboratory Standards Institute; 2019.

23. Wiese AD, Griffin Zhu, Mitchel EF, Grijalva CG. Changes in empyema among U.S. children in the pneumococcal conjugate vaccine era vaccine. 2016; 34 (50): 6243-6249.

24. López EL, Glatstein E, Ezcurra GC, Iacono M, Teplitz, Garnero AV et al. Rapid decrease in rates of hospitalization resulting from invasive pneumococcal disease and community-acquired pneumonia in children aged $<60$ months after 13-valent pneumococcal conjugate vaccine introduction in Argentina. J Pediatric Infect Dis Soc. 2018; 7 (1): 30-35. doi: 10.1093/jpids/piw089.

25. Olarte L, Barson WJ, Barson RM, Romero JR, Bradley JS, Tan TQ, Givner LB et al. Pneumococcal pneumonia requiring hospitalization in US children in the 13-valent pneumococcal conjugate vaccine. Clin Infect Dis. 2017; 64 (12): 1699-1704. doi: 10.1093/cid/cix115.

26. Kaplan SL, Barson WJ, Lin PL, Romero JR, Bradley JS, Tan TQ et al. Invasive pneumococcal disease in children's hospitals: 2014-2017. Pediatrics. 2019; 144 (3). pii: e20190567. doi: 10.1542/peds.2019-0567.

27. Villena GV, Cases VE, Fernández VA, de Pablo GA, Pérez RE, Porcel PJM et al. Recommendations of diagnosis and treatment of pleural effusion. Update. Arch Bronconeumol. $2014 ; 50$ (6): 235-249.
28. Machado KM, Pereira V, Coedo V, Arana M, Assandri E, Sisto $\mathrm{G}$ et al. Características epidemiológicas y clínicas de niños hospitalizados por enfermedad tuberculosa: Centro Hospitalario Pereira Rossell (2010-2013). Rev Méd Urug. 2015; 31 (3): 172-178.

29. Holcberg M, Zabala C, Gutierrez S, Sisto G, Sosa M, Gustavo G. Prevalencia y características clínico-epidemiológicas de los niños con tuberculosis diagnosticados a partir de un caso índice. Uruguay 2012-2014. Arch Pediatr Urugua. 2016; 87 (S1): S3-S10.

30. Amaya G, Badía F, Sisto G, Pujadas M, Pírez MC. Guía Nacional para el manejo de la Tuberculosis en situaciones especiales, Uruguay, año 2017. Capítulo: tuberculosis en la edad pediátrica. OPS-OMS Uruguay 2018. Disponible en: https://www.paho.org/uru/index.php?option=com_co ntent\&view=article\&id=1311: nueva-guia-nacional-parael-manejo-de-tuberculosis-en-uruguay $2017 \&$ Itemid $=310$. http://www.chlaep.org.uy/descargas/guia-nacionaltuberculosis2017digitales.pdf.

Financiamiento: Ninguno.

Conflicto de intereses: Ninguno.

Correspondencia:

Prof. María Catalina Pírez

E-mail:mcpirez@yahoo.com 